Publ. Mat. 62 (2018), 285-300

DOI: 10.5565 /PUBLMAT6211813

\title{
INFINITE SERIES IDENTITIES INVOLVING QUADRATIC AND CUBIC HARMONIC NUMBERS
}

\author{
Xiaoyuan Wang and Wenchang Chu
}

\begin{abstract}
By means of the modified Abel lemma on summation by parts, we investigate infinite series involving quadratic and cubic harmonic numbers. Several infinite series identities are established for $\pi^{2}$ and $\zeta(3)$ as consequences.
\end{abstract}

2010 Mathematics Subject Classification: Primary: 11M06; Secondary: 40A25.

Key words: Riemann zeta function, harmonic numbers, Abel's lemma on summation by parts, partial fraction decomposition, telescoping method.

\section{Introduction and preliminaries}

For two positive real numbers $a$ and $b$, define the following two sequences by

$$
h_{n}(a, b):=\sum_{k=1}^{n} \frac{1}{a k-a+b} \quad \text { and } \quad \bar{h}_{n}(a, b):=\sum_{k=1}^{n} \frac{(-1)^{k}}{a k-a+b} .
$$

They contain the well-known classical harmonic-like numbers as particular cases:

$$
\begin{aligned}
& H_{n}:=h_{n}(1,1)=\sum_{k=1}^{n} \frac{1}{k} \quad \text { and } \quad O_{n}:=h_{n}(2,1)=\sum_{k=1}^{n} \frac{1}{2 k-1} ; \\
& \bar{H}_{n}:=\bar{h}_{n}(1,1)=\sum_{k=1}^{n} \frac{(-1)^{k}}{k} \quad \text { and } \quad \bar{O}_{n}:=\bar{h}_{n}(2,1)=\sum_{k=1}^{n} \frac{(-1)^{k}}{2 k-1} .
\end{aligned}
$$

During the last two decades there has been growing interest in finding closed formulae of finite sums involving harmonic numbers. There are several approaches to evaluate these sums. By applying derivative operator to the known binomial identities and terminating hypergeometric formulae, Chu and De Donno $[\mathbf{9}, \mathbf{1 1}, \mathbf{1 2}]$ established numerous summation formulae. Boyadzhiev [2] evaluated several finite sums by the Euler transformation. Cheon and El-Mikkawy [4] studied these sums by expressing harmonic numbers in terms of the Stirling numbers of the first kind (cf. Comtet $[\mathbf{1 3}, \S 5.6]$ ). Flajolet and Salvy [15] developed a very 
powerful method, based on the residue technique, for the summation of series involving harmonic numbers. Wang [20] found further formulae concerning harmonic numbers by Riordan arrays. The most powerful method to investigate finite harmonic sums, "partial fraction decompositions", was introduced by Chu $[7,8]$ and subsequently developed by Ge-Liu-Luo [16] and Prodinger [18].

Instead, evaluation of infinite series containing harmonic numbers can be dated back to Euler, who discovered several surprising identities related to the Riemann zeta function. Three beautiful examples can be reproduced as follows (cf. $[\mathbf{1}, \mathbf{6}, \mathbf{1 4}])$ :

$$
2 \zeta(3)=\sum_{k \geq 1} \frac{H_{k}}{k^{2}}, \quad \frac{17 \pi^{4}}{360}=\sum_{k \geq 1} \frac{H_{k}^{2}}{k^{2}}, \quad \frac{\pi^{4}}{32}=\sum_{k \geq 1} \frac{O_{k}^{2}}{k^{2}} .
$$

In 1991, De Doelder [14] found numerous formulae by evaluating the digamma function. Borwein brothers [1] reviewed some identities by combining generating functions with Parseval's identity about the Fourier series in 1995. In 1997, Chu [6] devised a hypergeometric method by examining the coefficient of a monomial across known hypergeometric series equalities, that leads to a systematic investigation of the infinite series expressible in the Riemann zeta function (cf. $[\mathbf{3}, \mathbf{5}, \mathbf{1 7}, \mathbf{2 1}]$ ). Sofo and Hassani [19] derived further formulae by logarithmic integrals. Recently, Abel's lemma on summation by parts has been modified by $\mathrm{Chu}[\mathbf{1 0}]$ to derive several infinite series identities involving the classical harmonic numbers and their variants. The objective of the present work is to explore its further applications to infinite series involving quadratic and cubic harmonic numbers. Several remarkable formulae will be established. Here we highlight two infinite series expressions for $\pi^{2}$ (see Equations (9) and (11))

$$
\begin{aligned}
& \frac{\pi^{2}}{24}-\frac{3}{16}=\sum_{k \geq 1} \frac{H_{k}^{2}}{(k+1)(k+2)(k+3)}, \\
& \frac{\pi^{2}}{256}-\frac{1}{96}=\sum_{k \geq 1} \frac{O_{k}^{2}}{(2 k+1)(2 k+3)(2 k+5)} ;
\end{aligned}
$$

and two other ones for $\zeta(3)$ (see Equations (32) and (34))

$$
\begin{aligned}
& \frac{6 \zeta(3)+19}{12}=\sum_{k \geq 2} \frac{(2 k+1) H_{k}^{3}}{k(k+1)(k+2)(k-1)}, \\
& \frac{7 \zeta(3)-4}{512}=\sum_{k \geq 1} \frac{k O_{k}^{3}}{(2 k-3)(2 k-1)(2 k+1)(2 k+3)} .
\end{aligned}
$$


For subsequent applications, we reproduce Abel's lemma on summation by parts as follows. With an arbitrary complex sequence $\left\{\tau_{k}\right\}$, define the backward and forward difference operators $\nabla$ and $\Delta$, respectively, by

$$
\nabla \tau_{k}=\tau_{k}-\tau_{k-1} \quad \text { and } \quad \Delta \tau_{k}=\tau_{k}-\tau_{k+1},
$$

where $\Delta$ is adopted for convenience in the present paper, which differs from the usual operator $\Delta$ only in the minus sign. Then Abel's lemma on summation by parts (see [10] for a proof) may be reformulated as

$$
\Omega:=\sum_{k \geq 1} B_{k} \nabla A_{k}=[A B]_{+}-A_{0} B_{1}+\sum_{k \geq 1} A_{k} \Delta B_{k}
$$

provided that one of both series is convergent and the following limit exists:

$$
[A B]_{+}=\lim _{n \rightarrow \infty} A_{n} B_{n+1} .
$$

Under certain convergence conditions, this lemma can successively be applied. In the next section, we shall prove, by employing (2) twice, two main transformation theorems concerning the quadratic harmonic numbers $h_{k}^{2}(a, b)$ and $\bar{h}_{k}^{2}(a, b)$, that will yield several interesting identities for $\pi^{2}, \ln 2$, and the Catalan constant $G$. Then in Section 3, we shall establish, by applying (2) thrice, yet another general transformation theorem on the cubic harmonic numbers $h_{k}^{3}(a, b)$, from which we shall derive further two infinite series expressions for $\pi^{2}$ and two for $\zeta(3)$. In order to ensure the accuracy, all the formulae displayed in this paper have been checked numerically by appropriately devised Mathematica commands.

\section{Infinite series involving quadratic harmonic numbers}

For the $\Omega$-sum in (2), if there exist another difference pair $\left\{A_{k}^{\prime}, B_{k}^{\prime}\right\}$ and a constant $\beta$ such that

$$
\Omega^{\prime}:=\sum_{k \geq 1} A_{k} \Delta B_{k}=\beta+\sum_{k \geq 1} B_{k}^{\prime} \nabla A_{k}^{\prime},
$$

then we can reformulate the $\Omega^{\prime}$-sum further by (2) as follows:

$$
\Omega^{\prime}=\sum_{k \geq 1} A_{k} \Delta B_{k}=\beta+\left[A^{\prime} B^{\prime}\right]_{+}-A_{0}^{\prime} B_{1}^{\prime}+\sum_{k \geq 1} A_{k}^{\prime} \Delta B_{k}^{\prime} .
$$

Substituting this $\Omega^{\prime}$ into (2), we find, after having applied twice the modified Abel lemma on summation by parts, the following transformation formula. 
Lemma 1. For the two difference pairs $\left\{A_{k}, B_{k}\right\}$ and $\left\{A_{k}^{\prime}, B_{k}^{\prime}\right\}$ satisfying

$$
\sum_{k \geq 1} A_{k} \Delta B_{k}=\beta+\sum_{k \geq 1} B_{k}^{\prime} \nabla A_{k}^{\prime}
$$

the following infinite series formula holds

(5) $\Omega:=\sum_{k \geq 1} B_{k} \nabla A_{k}=\beta+[A B]_{+}+\left[A^{\prime} B^{\prime}\right]_{+}-A_{0} B_{1}-A_{0}^{\prime} B_{1}^{\prime}+\sum_{k \geq 1} A_{k}^{\prime} \Delta B_{k}^{\prime}$

provided that the both limits $[A B]_{+}$and $\left[A^{\prime} B^{\prime}\right]_{+}$exist and one of the two series displayed in (5) is convergent.

This lemma is more general than what we actually need. In fact, all the difference pairs $\left\{A_{k}, B_{k}\right\}$ and $\left\{A_{k}^{\prime}, B_{k}^{\prime}\right\}$ in this section satisfy, instead of (4), the following simpler condition $A_{k} \Delta B_{k}=B_{k}^{\prime} \nabla A_{k}^{\prime}$. Now we are in position to prove two main theorems, that will lead, as particular cases, to several infinite series identities containing quadratic harmonic numbers.

Theorem 2 (Two infinite series identities).

$$
\begin{aligned}
& \sum_{k \geq 1} \frac{a^{2} h_{k}^{2}(a, b)}{(a k+b)(a k+a+b)}=\frac{1}{a b}+\sum_{k \geq 0} \frac{1}{(a k+b)^{2}} \\
& \sum_{k \geq 1} \frac{4 a^{3} h_{k}^{2}(a, b)}{(a k+b)(a k+a+b)(a k+2 a+b)}=\sum_{k \geq 0} \frac{1}{(a k+b)^{2}}-\frac{a+2 b}{2 a b(a+b)} .
\end{aligned}
$$

Proof: Let $\left\{B_{k}, B_{k}^{\prime}\right\}$ be the two sequences

$$
B_{k}:=h_{k}^{2}(a, b) \quad \text { and } \quad B_{k}^{\prime}:=h_{k}(a, b)+h_{k+1}(a, b)
$$

with their differences being determined by

$$
\begin{aligned}
\Delta B_{k} & =-\frac{h_{k}(a, b)+h_{k+1}(a, b)}{a k+b}, \\
\Delta B_{k}^{\prime} & =-\frac{1}{a k+b}-\frac{1}{a k+a+b} .
\end{aligned}
$$

In order to be able to apply Lemma 1 , we will consider couples of sequences $\left\{A_{k}, A_{k}^{\prime}\right\}$ satisfying the property $A_{k} \Delta B_{k}=B_{k}^{\prime} \nabla A_{k}^{\prime}$ which is stronger than the condition (4).

First, it is almost trivial to verify that the two sequences $\left\{A_{k}, A_{k}^{\prime}\right\}$ given below satisfy the condition of Lemma 1

$$
A_{k}:=\frac{-a}{a k+a+b} \quad \text { and } \quad A_{k}^{\prime}:=\frac{-1}{a k+a+b} ;
$$


in view of their differences

$$
\begin{aligned}
& \nabla A_{k}=\frac{a^{2}}{(a k+b)(a k+a+b)}, \\
& \nabla A_{k}^{\prime}=\frac{a}{(a k+b)(a k+a+b)}
\end{aligned}
$$

as well as the limiting relations

$$
\begin{aligned}
& {[A B]_{+}=0, \quad A_{0} B_{1}=\frac{-a}{b^{2}(a+b)}} \\
& {\left[A^{\prime} B^{\prime}\right]_{+}=0, \quad A_{0}^{\prime} B_{1}^{\prime}=\frac{-2 a-3 b}{b(a+b)^{2}} .}
\end{aligned}
$$

Writing explicitly the equality corresponding to (5) and then simplifying the result, we get the first formula (6).

The second formula (7) can be shown analogously by choosing another couple of sequences $\left\{A_{k}, A_{k}^{\prime}\right\}$ by

$$
\begin{aligned}
& A_{k}:=\frac{-2 a}{(a k+a+b)(a k+2 a+b)}, \\
& A_{k}^{\prime}:=\frac{-1}{(a k+a+b)(a k+2 a+b)}
\end{aligned}
$$

and then computing their differences

$$
\begin{aligned}
& \nabla A_{k}=\frac{4 a^{2}}{(a k+b)(a k+a+b)(a k+2 a+b)} \\
& \nabla A_{k}^{\prime}=\frac{2 a}{(a k+b)(a k+a+b)(a k+2 a+b)}
\end{aligned}
$$

as well the limiting relations

$$
\begin{aligned}
& {[A B]_{+}=0, \quad A_{0} B_{1}=\frac{-2 a}{b^{2}(a+b)(2 a+b)}} \\
& {\left[A^{\prime} B^{\prime}\right]_{+}=0, \quad A_{0}^{\prime} B_{1}^{\prime}=\frac{-2 a-3 b}{b(a+b)^{2}(2 a+b)} .}
\end{aligned}
$$

The following interesting infinite series identities containing quadratic harmonic numbers can be easily deduced from Theorem 2 . 
Corollary $3(a=b=1$ in Theorem 2$)$.

$$
\begin{aligned}
& \sum_{k \geq 1} \frac{H_{k}^{2}}{(k+1)(k+2)}=1+\frac{\pi^{2}}{6}, \\
& \sum_{k \geq 1} \frac{H_{k}^{2}}{(k+1)(k+2)(k+3)}=\frac{\pi^{2}}{24}-\frac{3}{16} .
\end{aligned}
$$

Corollary 4 ( $a=2$ and $b=1$ in Theorem 2).

$$
\begin{aligned}
& \sum_{k \geq 1} \frac{O_{k}^{2}}{(2 k+1)(2 k+3)}=\frac{1}{8}+\frac{\pi^{2}}{32} \\
& \sum_{k \geq 1} \frac{O_{k}^{2}}{(2 k+1)(2 k+3)(2 k+5)}=\frac{\pi^{2}}{256}-\frac{1}{96} .
\end{aligned}
$$

Theorem 5 (Five infinite series identities).

$$
\sum_{k \geq 1} \frac{2 a^{2} \bar{h}_{k}^{2}(a, b)}{(a k+a+b)(a k+2 a+b)}=\sum_{k \geq 0} \frac{1}{(a k+b)^{2}}-\frac{3 a+2 b}{2 a b(a+b)}
$$

$$
\sum_{k \geq 1} \frac{2 a^{2} \bar{h}_{k}^{2}(a, b)}{(a k-2 a+b)(a k-3 a+b)}=\frac{5 a-2 b}{2 a(a-b)(2 a-b)}+\sum_{k \geq 0} \frac{1}{(a k+b)^{2}}
$$

(14) $\sum_{k \geq 1} \frac{a^{2}(3 a k+2 a+3 b) \bar{h}_{k}^{2}(a, b)}{(a k+b)(a k+a+b)(a k+2 a+b)}=2\left\{\sum_{k \geq 0} \frac{(-1)^{k}}{a k+b}\right\}^{2}-\frac{1}{2(a+b) b}$,

$$
\begin{aligned}
& \sum_{k \geq 1} \frac{(-1)^{k} 4 a^{2} \bar{h}_{k}^{2}(a, b)}{(a k+b)(a k+2 a+b)}=\frac{1}{2 b(a+b)}-\sum_{k \geq 0} \frac{(-1)^{k}}{(a k+b)^{2}} \\
& \sum_{k \geq 1} \frac{(-1)^{k} a(2 a k+a+2 b) \bar{h}_{k}^{2}(a, b)}{(a k+b)(a k+a+b)}=\frac{1}{a b}-\sum_{k \geq 0} \frac{(-1)^{k}}{(a k+b)^{2}}-\frac{2}{a} \sum_{k \geq 0} \frac{(-1)^{k}}{a k+b}
\end{aligned}
$$

Proof: Following the proof of Theorem 2, we first fix the sequences $\left\{B_{k}, B_{k}^{\prime}\right\}$ by

$$
B_{k}:=\bar{h}_{k}^{2}(a, b) \quad \text { and } \quad B_{k}^{\prime}:=\bar{h}_{k}(a, b)+\bar{h}_{k+1}(a, b)
$$


and determine their differences by

$$
\begin{aligned}
\Delta B_{k} & =\frac{(-1)^{k}}{a k+b}\left\{\bar{h}_{k}(a, b)+\bar{h}_{k+1}(a, b)\right\}, \\
\Delta B_{k}^{\prime} & =\frac{(-1)^{k}}{a k+b}-\frac{(-1)^{k}}{a k+a+b} .
\end{aligned}
$$

To prove Theorem 5, it suffices to figure out the difference couples $\left\{A_{k}, A_{k}^{\prime}\right\}$ according to Lemma 1 . They are sketched below so that the reader will not have difficulty to follow the approach, even though the final confirmations for these identities are made by combining partial fraction decompositions and the telescoping method.

Formula (12): Sequence couple

$$
A_{k}:=\frac{2}{a k+2 a+b} \quad \text { and } \quad A_{k}^{\prime}=\frac{(-1)^{k}}{(a k+a+b)(a k+2 a+b)} ;
$$

with their differences

$$
\begin{aligned}
& \nabla A_{k}=\frac{-2 a}{(a k+a+b)(a k+2 a+b)}, \\
& \nabla A_{k}^{\prime}=\frac{2(-1)^{k}}{(a k+b)(a k+2 a+b)}
\end{aligned}
$$

as well as limiting relations

$$
\begin{aligned}
& {[A B]_{+}=0, \quad A_{0} B_{1}=\frac{2}{b^{2}(2 a+b)}} \\
& {\left[A^{\prime} B^{\prime}\right]_{+}=0, \quad A_{0}^{\prime} B_{1}^{\prime}=\frac{-1}{b(a+b)^{2}} .}
\end{aligned}
$$

Formula (13): Sequence couple

$$
A_{k}:=\frac{2}{a k-2 a+b} \quad \text { and } \quad A_{k}^{\prime}:=\frac{(-1)^{k}}{(a k+b)(a k-a+b)} ;
$$

with their differences

$$
\begin{aligned}
& \nabla A_{k}=\frac{-2 a}{(a k-2 a+b)(a k-3 a+b)}, \\
& \nabla A_{k}^{\prime}=\frac{2(-1)^{k}}{(a k+b)(a k-2 a+b)}
\end{aligned}
$$


as well as limiting relations

$$
\begin{aligned}
& {[A B]_{+}=0, \quad A_{0} B_{1}=\frac{2}{b^{2}(b-2 a)}} \\
& {\left[A^{\prime} B^{\prime}\right]_{+}=0, \quad A_{0}^{\prime} B_{1}^{\prime}=\frac{2 a+b}{b^{2}\left(a^{2}-b^{2}\right)} .}
\end{aligned}
$$

Formula (14): Sequence couple

$$
A_{k}:=\frac{(a k+b)(2 a k+3 a+2 b)}{(a k+a+b)(a k+2 a+b)} \quad \text { and } \quad A_{k}^{\prime}:=\frac{(-1)^{k}}{a k+2 a+b} ;
$$

with their differences

$$
\begin{aligned}
& \nabla A_{k}=\frac{a^{2}(3 a k+2 a+3 b)}{(a k+b)(a k+a+b)(a k+2 a+b)}, \\
& \nabla A_{k}^{\prime}=\frac{(-1)^{k}(2 a k+3 a+2 b)}{(a k+a+b)(a k+2 a+b)} ;
\end{aligned}
$$

as well as limiting relations

$$
\begin{array}{ll}
{[A B]_{+}=2\left\{\sum_{k \geq 0} \frac{(-1)^{k}}{a k+b}\right\}^{2},} & A_{0} B_{1}=\frac{3 a+2 b}{b(a+b)(2 a+b)} ; \\
{\left[A^{\prime} B^{\prime}\right]_{+}=0,} & A_{0}^{\prime} B_{1}^{\prime}=\frac{-1}{b(a+b)} .
\end{array}
$$

Formula (15): Sequence couple

$$
\begin{aligned}
& A_{k}:=\frac{(-1)^{k-1} 2 a}{(a k+a+b)(a k+2 a+b)}, \\
& A_{k}^{\prime}:=\frac{1}{(a k+a+b)(a k+2 a+b)}
\end{aligned}
$$

with their differences

$$
\begin{aligned}
& \nabla A_{k}=\frac{(-1)^{k-1} 4 a}{(a k+b)(a k+2 a+b)}, \\
& \nabla A_{k}^{\prime}=\frac{-2 a}{(a k+b)(a k+a+b)(a k+2 a+b)}
\end{aligned}
$$


as well as limiting relations

$$
\begin{aligned}
& {[A B]_{+}=0, \quad A_{0} B_{1}=\frac{-2 a}{b^{2}(a+b)(2 a+b)} ;} \\
& {\left[A^{\prime} B^{\prime}\right]_{+}=0, \quad A_{0}^{\prime} B_{1}^{\prime}=\frac{-1}{b(a+b)^{2}} .}
\end{aligned}
$$

Formula (16): Sequence couple

$$
A_{k}:=\frac{(-1)^{k-1} a}{a k+a+b} \quad \text { and } \quad A_{k}^{\prime}:=\frac{1}{a k+a+b} ;
$$

with their differences

$$
\begin{aligned}
& \nabla A_{k}=\frac{(-1)^{k-1} a(2 a k+a+2 b)}{(a k+b)(a k+a+b)}, \\
& \nabla A_{k}^{\prime}=\frac{-a}{(a k+b)(a k+a+b)} ;
\end{aligned}
$$

as well as limiting relations

$$
\begin{aligned}
& {[A B]_{+}=0, \quad A_{0} B_{1}=\frac{-a}{b^{2}(a+b)}} \\
& {\left[A^{\prime} B^{\prime}\right]_{+}=0, \quad A_{0}^{\prime} B_{1}^{\prime}=\frac{-2 a-b}{b(a+b)^{2}} .}
\end{aligned}
$$

As examples of Theorem 5, we record ten remarkable infinite series identities.

Corollary $6(a=b=1$ in Theorem 5$)$.

$$
\begin{aligned}
& \sum_{k \geq 1} \frac{\bar{H}_{k}^{2}}{(k+2)(k+3)}=\frac{\pi^{2}}{12}-\frac{5}{8}, \\
& \sum_{k \geq 3} \frac{\bar{H}_{k}^{2}}{(k-1)(k-2)}=\frac{\pi^{2}}{12}-\frac{1}{4}, \\
& \sum_{k \geq 1} \frac{(3 k+5) \bar{H}_{k}^{2}}{(k+1)(k+2)(k+3)}=2(\ln 2)^{2}-\frac{1}{4}, \\
& \sum_{k \geq 1} \frac{(-1)^{k} \bar{H}_{k}^{2}}{(k+1)(k+3)}=\frac{1}{16}-\frac{\pi^{2}}{48}, \\
& \sum_{k \geq 1} \frac{(-1)^{k}(2 k+3) \bar{H}_{k}^{2}}{(k+1)(k+2)}=1-\frac{\pi^{2}}{12}-2 \ln 2 .
\end{aligned}
$$


Corollary 7 ( $a=2$ and $b=1$ in Theorem 5$)$.

$$
\begin{aligned}
& \sum_{k \geq 1} \frac{\bar{O}_{k}^{2}}{(2 k+3)(2 k+5)}=\frac{\pi^{2}}{64}-\frac{1}{12}, \\
& \sum_{k \geq 1} \frac{\bar{O}_{k}^{2}}{(2 k-5)(2 k-3)}=\frac{\pi^{2}}{64}+\frac{1}{12}, \\
& \sum_{k \geq 1} \frac{(6 k+7) \bar{O}_{k}^{2}}{(2 k+1)(2 k+3)(2 k+5)}=\frac{\pi^{2}}{32}-\frac{1}{24}, \\
& \sum_{k \geq 1} \frac{(-1)^{k} \bar{O}_{k}^{2}}{(2 k+1)(2 k+5)}=\frac{1}{96}-\frac{G}{16}, \\
& \sum_{k \geq 1} \frac{(-1)^{k}(k+1) \bar{O}_{k}^{2}}{(2 k+1)(2 k+3)}=\frac{1}{16}-\frac{G}{8}-\frac{\pi}{32} .
\end{aligned}
$$

In the last two series, we have used $G$ to denote the Catalan constant:

$$
G=\sum_{k \geq 0}(-1)^{k} /(2 k+1)^{2}=0.9159 \ldots
$$

\section{Infinite series involving cubic harmonic numbers}

Under the same condition of Lemma 1, we may apply (2) further. Let $\left\{A_{k}^{\prime \prime}, B_{k}^{\prime \prime}\right\}$ be the third difference pair subject to the condition

$$
\Omega^{\prime \prime}:=\sum_{k \geq 1} A_{k}^{\prime} \Delta B_{k}^{\prime}=\rho+\sum_{k \geq 1} B_{k}^{\prime \prime} \nabla A_{k}^{\prime \prime},
$$

where $\rho$ is a constant. Then we can manipulate the $\Omega^{\prime \prime}$-sum again by (2) in the following manner:

$$
\Omega^{\prime \prime}=\sum_{k \geq 1} A_{k}^{\prime} \Delta B_{k}^{\prime}=\rho+\left[A^{\prime \prime} B^{\prime \prime}\right]_{+}-A_{0}^{\prime \prime} B_{1}^{\prime \prime}+\sum_{k \geq 1} A_{k}^{\prime \prime} \Delta B_{k}^{\prime \prime} .
$$

Substituting this $\Omega^{\prime \prime}$ into (5), we find, after having applied thrice the modified Abel lemma on summation by parts, another transformation formula.

Lemma 8. For the three difference pairs $\left\{A_{k}, B_{k}\right\},\left\{A_{k}^{\prime}, B_{k}^{\prime}\right\}$, and $\left\{A_{k}^{\prime \prime}, B_{k}^{\prime \prime}\right\}$ satisfying (4) and

$$
\sum_{k \geq 1} A_{k}^{\prime} \Delta B_{k}^{\prime}=\rho+\sum_{k \geq 1} B_{k}^{\prime \prime} \nabla A_{k}^{\prime \prime}
$$


the following infinite series formula holds

$$
\begin{aligned}
\Omega:=\sum_{k \geq 1} B_{k} \nabla A_{k} & =[A B]_{+}+\left[A^{\prime} B^{\prime}\right]_{+}+\left[A^{\prime \prime} B^{\prime \prime}\right]_{+} \\
& +\beta+\rho-A_{0} B_{1}-A_{0}^{\prime} B_{1}^{\prime}-A_{0}^{\prime \prime} B_{1}^{\prime \prime}+\sum_{k \geq 1} A_{k}^{\prime \prime} \Delta B_{k}^{\prime \prime}
\end{aligned}
$$

provided that the three limits $[A B]_{+},\left[A^{\prime} B^{\prime}\right]_{+}$, and $\left[A^{\prime} B^{\prime}\right]_{+}$exist, and one of the two series displayed in (28) is convergent.

By employing this lemma, we can prove the following general theorem.

Theorem 9 (Two infinite series identities).

$$
\begin{aligned}
\sum_{k \geq 1} \frac{2 a^{3} h_{k}^{3}(a, b)}{(2 a k+a+2 b)(2 a k-3 a+2 b)}=\frac{4}{a(2 b-a)} & +\sum_{k \geq 0} \frac{1}{(a k+b)^{2}} \\
\sum_{k \geq 1} \frac{2 a^{3}(2 a k-a+2 b) h_{k}^{3}(a, b)}{(a k+b)(a k+a+b)(a k-a+b)(a k-2 a+b)} & =\frac{1}{a b(b-a)} \\
& +\sum_{k \geq 0} \frac{1}{(a k+b)^{3}}
\end{aligned}
$$

Proof: Similar to the preceding proofs, we shall fix the sequences $\left\{B_{k}\right.$, $\left.B_{k}^{\prime}, B_{k}^{\prime \prime}\right\}$ by

$$
\begin{aligned}
B_{k} & :=h_{k}^{3}(a, b), \\
B_{k}^{\prime} & :=h_{k}^{2}(a, b)+h_{k}(a, b) h_{k+1}(a, b)+h_{k+1}^{2}(a, b), \\
B_{k}^{\prime \prime} & :=h_{k+1}(a, b) ;
\end{aligned}
$$

with their differences being given by

$$
\begin{aligned}
\Delta B_{k} & =\frac{h_{k}^{2}(a, b)+h_{k}(a, b) h_{k+1}(a, b)+h_{k+1}^{2}(a, b)}{-(a k+b)}, \\
\Delta B_{k}^{\prime} & =\frac{a(2 a k+a+2 b)}{(a k+b)^{2}(a k+a+b)^{2}}-\frac{3(2 a k+a+2 b) h_{k+1}(a, b)}{(a k+b)(a k+a+b)}, \\
\Delta B_{k}^{\prime \prime} & =\frac{-1}{a k+a+b} .
\end{aligned}
$$


Then the first formula (29) can be confirmed by specifying in Lemma 8 the triple sequences $\left\{A_{k}, A_{k}^{\prime}, A_{k}^{\prime \prime}\right\}$ by

$$
\begin{aligned}
A_{k} & :=\frac{-(a k+b)}{a(2 a k+a+2 b)(2 a k-a+2 b)}, \\
A_{k}^{\prime} & :=\frac{-1}{2 a^{2}(2 a k+a+2 b)}, \\
A_{k}^{\prime \prime} & :=\frac{-3}{2 a^{3}(a k+a+b)} ;
\end{aligned}
$$

and evaluating their differences

$$
\begin{aligned}
\nabla A_{k} & =\frac{1}{(2 a k+a+2 b)(2 a k-3 a+2 b)}, \\
\nabla A_{k}^{\prime} & =\frac{1}{a(2 a k+a+2 b)(2 a k-a+2 b)}, \\
\nabla A_{k}^{\prime \prime} & =\frac{3}{2 a^{3}(a k+b)(a k+a+b)} ;
\end{aligned}
$$

as well as limiting relations

$$
\begin{aligned}
& {[A B]_{+}=0, \quad A_{0} B_{1}=\frac{1}{a b^{2}\left(a^{2}-4 b^{2}\right)} ;} \\
& {\left[A^{\prime} B^{\prime}\right]_{+}=0, \quad A_{0}^{\prime} B_{1}^{\prime}=\frac{-\left(3 a^{2}+9 a b+7 b^{2}\right)}{2 a^{2} b^{2}(a+b)^{2}(a+2 b)} ;} \\
& {\left[A^{\prime \prime} B^{\prime \prime}\right]_{+}=0, \quad A_{0}^{\prime \prime} B_{1}^{\prime \prime}=\frac{-3(a+2 b)}{2 a^{3} b(a+b)^{2}} .}
\end{aligned}
$$

However, one should be careful about the constants $\beta=0$ and $\rho$, which can be evaluated by combining partial fractions with the telescoping method:

$$
\begin{aligned}
\rho= & \frac{-1}{2 a} \sum_{k \geq 1} \frac{1}{(a k+b)^{2}(a k+a+b)^{2}} \\
= & \sum_{k \geq 1}\left\{\frac{1}{a^{4}(a k+b)}-\frac{1}{a^{4}(a k+a+b)}\right\} \\
& -\sum_{k \geq 1}\left\{\frac{1}{2 a^{3}(a k+b)^{2}}+\frac{1}{2 a^{3}(a k+a+b)^{2}}\right\} \\
= & \frac{a+2 b}{2 a^{4}(a+b)^{2}}-\frac{1}{a^{3}} \sum_{k \geq 1} \frac{1}{(a k+a+b)^{2}} .
\end{aligned}
$$


As for the second formula (30), it can be done by letting alternatively in Lemma 8 the triple sequences $\left\{A_{k}, A_{k}^{\prime}, A_{k}^{\prime \prime}\right\}$ by

$$
\begin{aligned}
A_{k} & :=\frac{-1}{a(a k-a+b)(a k+a+b)}, \\
A_{k}^{\prime} & :=\frac{-1}{2 a^{2}(a k+b)(a k+a+b)}, \\
A_{k}^{\prime \prime} & :=\frac{-3}{2 a^{3}(a k+a+b)^{2}}
\end{aligned}
$$

and then determining their differences

$$
\begin{aligned}
\nabla A_{k} & =\frac{2 a k-a+2 b}{(a k+b)(a k+a+b)(a k-a+b)(a k-2 a+b)}, \\
\nabla A_{k}^{\prime} & =\frac{1}{a(a k+b)(a k+a+b)(a k-a+b)}, \\
\nabla A_{k}^{\prime \prime} & =\frac{3(2 a k+a+2 b)}{2 a^{2}(a k+b)^{2}(a k+a+b)^{2}} ;
\end{aligned}
$$

as well as the limiting relations

$$
\begin{aligned}
& {[A B]_{+}=0, \quad A_{0} B_{1}=\frac{1}{a b^{3}\left(a^{2}-b^{2}\right)} ;} \\
& {\left[A^{\prime} B^{\prime}\right]_{+}=0, \quad A_{0}^{\prime} B_{1}^{\prime}=\frac{-\left(3 a^{2}+9 a b+7 b^{2}\right)}{2 a^{2} b^{3}(a+b)^{3}} ;} \\
& {\left[A^{\prime \prime} B^{\prime \prime}\right]_{+}=0, \quad A_{0}^{\prime \prime} B_{1}^{\prime \prime}=\frac{-3(a+2 b)}{2 a^{3} b(a+b)^{3}} .}
\end{aligned}
$$

Also in this case, we have $\beta=0$ and another non vanishing constant

$$
\begin{aligned}
\rho= & \frac{-1}{2 a} \sum_{k \geq 1} \frac{2 a k+a+2 b}{(a k+b)^{3}(a k+a+b)^{3}} \\
= & \sum_{k \geq 1}\left\{\frac{1}{2 a^{4}(a k+b)^{2}}-\frac{1}{2 a^{4}(a k+a+b)^{2}}\right\} \\
& -\sum_{k \geq 1}\left\{\frac{1}{2 a^{3}(a k+b)^{3}}+\frac{1}{2 a^{3}(a k+a+b)^{3}}\right\} \\
= & \frac{b}{2 a^{4}(a+b)^{3}}-\frac{1}{a^{3}} \sum_{k \geq 1} \frac{1}{(a k+a+b)^{3}} .
\end{aligned}
$$


As consequences of Theorem 9, we display below four infinite series identities involving cubic harmonic numbers.

Corollary $10(a=b=1$ in Theorem 9$)$.

$$
\begin{aligned}
& \sum_{k \geq 1} \frac{H_{k}^{3}}{(2 k-1)(2 k+3)}=\frac{24+\pi^{2}}{12}, \\
& \sum_{k \geq 2} \frac{(2 k+1) H_{k}^{3}}{k(k+1)(k+2)(k-1)}=\frac{6 \zeta(3)+19}{12} .
\end{aligned}
$$

Corollary $11(a=2$ and $b=1$ in Theorem 9).

$$
\begin{aligned}
& \sum_{k \geq 2} \frac{O_{k}^{3}}{(k+1)(k-1)}=\frac{26+\pi^{2}}{8}, \\
& \sum_{k \geq 1} \frac{k O_{k}^{3}}{(2 k-3)(2 k-1)(2 k+1)(2 k+3)}=\frac{7 \zeta(3)-4}{512} .
\end{aligned}
$$

Acknowledgement. The first author has been supported partially by Liaoning Educational Commission of China (No. JDL2016018) during this research.

\section{References}

[1] D. Borwein And J. M. Borwein, On an intriguing integral and some series related to $(4)$, Proc. Amer. Math. Soc. 123(4) (1995), 1191-1198. DOI: $10.2307 / 2160718$.

[2] K. N. BoyadzHIEv, Harmonic number identities via Euler's transform, J. Integer Seq. 12(6) (2009), Article 09.6.1, 8 pp.

[3] X. Chen And W. Chu, The Gauss ${ }_{2} F_{1}(1)$-summation theorem and harmonic number identities, Integral Transforms Spec. Funct. 20(12) (2009), 925-935. DOI: 10.1080/10652460903016166.

[4] G.-S. Cheon and M. E. A. El-Mikkawy, Generalized harmonic number identities and a related matrix representation, J. Korean Math. Soc. 44(2) (2007), 487-498. DOI: 10.4134/JKMS.2007.44.2. 487.

[5] J. Choi And H. M. Srivastava, Some summation formulas involving harmonic numbers and generalized harmonic numbers, Math. Comput. Modelling 54(9-10) (2011), 2220-2234. DOI: 10.1016/j. mcm. 2011.05.032. 
[6] W. CHu, Hypergeometric series and the Riemann zeta function, Acta Arith. 82(2) (1997), 103-118. DOI: 10.4064/aa-82-2-103-118.

[7] W. CHu, Harmonic number identities and Hermite-Padé approximations to the logarithm function, J. Approx. Theory 137(1) (2005), 42-56. DOI: 10.1016/j.jat.2005.07.008.

[8] W. CHu, Partial-fraction decompositions and harmonic number identities, J. Combin. Math. Combin. Comput. 60 (2007), 139-153.

[9] W. CHu, Summation formulae involving harmonic numbers, Filomat 26(1) (2012), 143-152. DOI: 10.2298/FIL1201143C.

[10] W. CHU, Infinite series identities on harmonic numbers, Results Math. 61(3-4) (2012), 209-221. DOI: 10.1007/s00025-010-0089-2.

[11] W. Chu And L. De Donno, Hypergeometric series and harmonic number identities, Adv. in Appl. Math. 34(1) (2005), 123-137. DOI: 10.1016/j . aam. 2004.05.003.

[12] W. Chu And L. De Donno, Identià binomiali e numeri armonici, Boll. Unione Mat. Ital. Sez. B Artic. Ric. Mat. (8) 10(1) (2007), 213-235.

[13] L. Comtet, "Advanced Combinatorics. The Art of Finite and Infinite Expansions", Revised and enlarged edition. D. Reidel Publishing Co., Dordrecht, 1974. DOI : 10.1007/978-94-010-2196-8.

[14] P. J. De Doelder, On some series containing $\psi(x)-\psi(y)$ and $(\psi(x)-\psi(y))^{2}$ for certain values of $x$ and $y$, J. Comput. Appl. Math. 37(1-3) (1991), 125-141. DOI : 10.1016/0377-0427 (91) 90112-W.

[15] P. Flajolet AND B. SAlvy, Euler sums and contour integral representations, Experiment. Math. 7(1) (1998), 15-35. DOI: 10.1080/10586458.1998.10504356.

[16] J.-K. GE, T.-T. LiU, AND Q.-M. Luo, A new identity via partial fraction decomposition, Filomat 30(7) (2016), 1957-1967. DOI: 10.2298/FIL1607957G.

[17] H. LiU And W. WAng, Harmonic number identities via hypergeometric series and Bell polynomials, Integral Transforms Spec. Funct. 23(1) (2012), 49-68. DOI: 10.1080/10652469.2011.553718.

[18] H. Prodinger, Identities involving harmonic numbers that are of interest for physicists, Util. Math. 83 (2010), 291-299.

[19] A. Sofo AND M. Hassani, Quadratic harmonic number sums, Appl. Math. E-Notes 12 (2012), 110-117.

[20] W. Wang, Riordan arrays and harmonic number identities, Comput. Math. Appl. 60(5) (2010), 1494-1509. DOI: 10.1016/j.camwa. 2010.06 .031$. 
[21] D.-Y. ZhenG, Further summation formulae related to generalized harmonic numbers, J. Math. Anal. Appl. 335(1) (2007), 692-706. DOI: $10.1016 / \mathrm{j} \cdot$ jmaa.2007.02.002.

Xiaoyuan Wang:

School of Science

Dalian Jiaotong University

Dalian 116028

P. R. China

E-mail address: xiaoyuanwang27@yahoo.com

Wenchang Chu:

School of Mathematics and Statistics

Zhoukou Normal University

Zhoukou (Henan)

P. R. China

E-mail address: chu.wenchang@unisalento.it

Primera versió rebuda el 21 de novembre de 2016, darrera versió rebuda el 20 d'abril de 2017. 